\title{
Molecular Characteristics and Antimicrobial Susceptibility Profile of Pseudomonas aeruginosa isolated from patients attending Healthcare Facilities in Mthatha, South Africa
}

Mojisola C. Hosu

Walter Sisulu University

Sandeep D. Vasaikar

Walter Sisulu University

Grace E. Okuthe

Walter Sisulu University

teke apalata ( $\square$ ruffinapalata@gmail.com )

Walter Sisulu University

Research

Keywords: P. aeruginosa, healthcare-associated infections, ESBL, MBL, blaSHV, blaTEM, blaCTX-M, Realtime PCR

Posted Date: February 28th, 2020

DOI: https://doi.org/10.21203/rs.3.rs-15431/v1

License: (c) (1) This work is licensed under a Creative Commons Attribution 4.0 International License.

Read Full License 


\section{Abstract}

Background Pseudomonas aeruginosa is a common pathogen causing healthcare-associated infections most especially in critically ill and immunocompromised patients. This pathogen poses a public health threat due to its innate resistance to many antimicrobial agents and its ability to acquire new resistance mechanisms under pressure. Infections with Extended spectrum $\beta$-lactamases (ESBL)-producing isolates result into outbreaks that lead to serious antibiotic management concerns with higher mortality and morbidity and significant economic causatives. In this study, we evaluated the antimicrobial resistance patterns and characterized genetically the ESBLs and Metallo- $\beta$-lactamases (MBL) produced by this pathogen.

Methods Isolates of $P$. aeruginosa cultured from patients who attended Nelson Mandela Academic Hospital and other clinics in the four district municipalities of the Eastern Cape between August 2017 and May 2019 were identified; and their antibiotic resistance patterns were tested against amikacin, aztreonam, cefepime, ceftazidime, ciprofloxacin, doripenem, gentamicin, imipenem, levofloxacin, meropenem, piperacillin, piperacillin/tazobactam and tobramycin using the bioMérieux VITEK® 2 and confirmed by Beckman autoSCAN-4 System. Real-time PCR was done using Roche Light Cycler 2.0 to detect the presence of ESBLs; blaSHV, blaTEM and blaCTX-M genes; and MBLs; blaIMP, blaVIM.

Results High antibiotic resistance in decreasing order was observed in piperacillin (64.2\%), aztreonam (57.8\%), cefepime (51.5\%), ceftazidime (51.0\%), piperacillin/tazobactam (50.5\%), and imipenem (46.6\%). A total of $75(36.8 \%)$ multidrug resistant (MDR) isolates were observed of the total pool of isolates. The blaTEM, blaSHV and blaCTX-M was detected in $79.3 \%, 69.5 \%$ and $31.7 \%$ isolates $(n=82)$, respectively. The blaIMP was detected in $1.25 \%$ while no blaVIM was detected in any of the isolates tested.

Conclusions The study showed a high rate of MDR P. aeruginosa in our setting. The vast majority of these resistant isolates carried blaTEM and blaSHV genes. Continuous monitoring of antimicrobial resistance and strict compliance towards infection prevention and control practices are the best defence against spread of MDR P. aeruginosa.

\section{Background}

P. aeruginosa is an opportunistic pathogen causing infections especially in immunocompromised patients. It is the leading cause of nosocomial infections such as urinary tract infections, surgical site infections, pneumonia, bacteremia and septicaemia [1-3] It is one of the ESKAPE pathogens that is most medically and epidemiologically significant and has been implicated as a principal cause of chronic lung infections in cystic fibrosis (CF) patients and severe infections in burn victims [4-7]. The World Health Organization (WHO) has categorized P. aeruginosa as a critical priority pathogen which needs urgent novel antibiotics intervention and has been given a serious threat level due to multidrug resistance displayed to many antibiotics $[8,9]$. The growing resistance of P. aeruginosa to several antibiotics, as a result of excessive antibiotic administration, has resulted to the accumulation of antibiotic resistance and 
cross-resistance between antibiotics and the advent of multidrug-resistant (MDR) forms of P. aeruginosa. P. aeruginosa infections are generally linked with high mortality; this is due to its innate resistance to several antimicrobial agents and acquired resistance via mutation and horizontal transfer $[10,11]$ Various mechanisms involved in the resistance of $P$. aeruginosa include over expression of efflux pump, acquisition of Extended-Spectrum $\beta$-Lactamases (ESBLs) and Metallo- $\beta$-Lactamases (MBLs) [12]. ESBLs are a cluster of $\beta$-lactamases that inactivates $\beta$-lactams especially oxymino- $\beta$-lactams and monobactams, and are repressed by $\beta$-lactamase inhibitors, such as clavulanic acid. They are encoded on plasmids and can easily be conveyed from one organism to another $[13,14]$. ESBLs were first discovered in 1983 and ever since over 500 diverse $\beta$-lactamases have been described to date $[15,16]$. ESBL enzymes according to Ambler classification are categorized into two, $A$ and $D$. The most prevalent enzymes in class $\mathrm{A}$ include bla $\mathrm{TEM}_{\mathrm{TEM}}, \mathrm{bla}_{\mathrm{CTX}-\mathrm{M}}$ and $\mathrm{bla}_{\mathrm{SHV}}$, and has been described in $\mathrm{P}$. aeruginosa strains $[13,17,18]$. The emergence of beta-lactamase enzymes is majorly due to chromosomal mutation and procurement of resistance genes which are moved about on various mobile genetic elements (MGEs) such as bacteriophages, genomic islands, integrons, plasmids and insertion sequences [19]. The production of these enzymes is a going concern for infection control supervision because it restricts therapeutic choices. Continuous monitoring and timely detection of ESBL and MBL producing organisms is critical to establish suitable antimicrobial therapy and to thwart their spread [16]. Polymerase chain reaction (PCR)-based methods are critical to establish the prevalence and characterization of beta lactamases due to the presence of multiple resistance genes in some microorganisms [20]. Real-time PCR detection of ESBL enhances faster diagnosis and timely management of epidemiological information for monitoring outbreak situations [21]. Several studies have documented the antimicrobial resistance in P. aeruginosa including genotypic detection of ESBLs and MBLs [21-25]. Studies on ESBL-producing P. aeruginosa in South Africa have also been documented [26-29] but scarce data exist in the Eastern Cape particularly in the former Transkei region on the molecular characteristics of ESBLs and MBLs in P. aeruginosa. Antibiotic surveillance studies are important for the design of control strategies for preventing bacterial resistance and establishing therapeutic guidelines as well as for a better understanding of bacterial epidemiology. The aim of this study was to examine the antimicrobial susceptibility profiles of clinical isolates of $P$. aeruginosa from Mthatha and surrounding districts and to investigate their ESBL resistance mechanisms.

\section{Materials And Methods}

Study Design

A prospective, descriptive study based on laboratory analysis. Samples from patients were collected from August 2016 to May 2019.

Study Setting

This was a laboratory analysis at the Microbiology Laboratory of the National Health Laboratory Services (NHLS) at Nelson Mandela Academic Hospital (NMAH) and the Department of Laboratory Medicine and 
Pathology, Faculty of Health Sciences, WSU. In this study, 204 non-duplicate isolates from patients were arbitrarily obtained from August 2016 to May 2019. The samples were drawn from four district municipalities of the Eastern Cape Province which include Alfred Nzo, Amathole, Joe Gqabi and Oliver Reginald (OR) Tambo district Municipalities. Health facilities include primary health centres/clinics, secondary district hospitals and a tertiary academic hospital located in Mthatha. Clinical samples were sent from various primary and secondary clinics in the afore- mentioned municipalities to Mthatha at the NHLS for analysis.

Specimen collection and analysis

Non-duplicate $P$. aeruginosa isolates were collected from Mthatha, other clinics and hospitals from the four district municipalities. Specimens included throat swabs, wound swabs, swabs from abscesses, sputum, urine, blood culture and catheter tips. Demographic characteristics of patients and medical histories were collected from medical records including date of specimen collection, gender, age, test ordered and hospital/clinic. All samples were routinely cultured on MacConkey and Blood agar plates. Blood and sputum were also cultured on chocolate agar. Suspected colonies were plated on Cetrimide agar and identified by gram staining, colony characteristics, motility, pyocyanin production and characteristics grape-like odour $(30,31)$. Strains were identified to the species level with Vitek $2 \mathrm{GN}$ (bioMérieux, Inc. USA) ID cards and confirmed by Microscan NID 2 panels (Beckman Coulter, Inc. USA).

Antimicrobial susceptibility

Antimicrobial susceptibility was obtained by determining MIC using Microscan dehydrated broth microdilution negative MIC Panel Type 44 (NM44) (Beckman Coulter, Inc. USA) following the manufacturer's guidelines [32,33] and Clinical Laboratory Standards Institute. MICs were interpreted following CLSI guidelines (M100-S27 breakpoints) [34]. The following antibiotics were tested in the panels: amikacin, aztreonam, cefepime, ceftazidime, ciprofloxacin, doripenem, gentamicin, imipenem, levofloxacin, meropenem, piperacillin/tazobactam, piperacillin and tobramycin.

Criterion for Multidrug resistance

The classification of MDR was performed according to Magiorakos et al., [35]. (MDR was defined as nonsusceptibility to at least one agent in three or more antimicrobial categories).

Molecular confirmation of strains and Real-Time PCR for identification

DNA extraction was done using Roche MagNA Pure Bacteria lysis buffer, MagNA Pure Compact Nucleic Acid Isolation kit and PCR grade water (Roche Applied Science, Indianapolis), following manufacturer's instructions.

Real time PCR was carried out in the Light Cycler 2.0 instrument (Roche Applied Science, Germany) using Fast start Light Cycler 480 Hybprobes Master kit (Roche Diagnostics, USA). Specific primers targeting the genes gyrB were amplified by singleplex rPCR using primers and probes shown in Table 1 and were used 
to confirm identity of the isolates. Primers were designed by TIB-Molbiol (Berlin, Germany). rPCR assay was performed according to previously published protocol [18]. Absolute quantification was carried out using the Light Cycler software 4.05. P. aeruginosa ATCC 27853 was used as a positive control.

Molecular ESBL and MBL Detection by Singleplex rPCR

Real-time PCR for $b / a_{\mathrm{CTX}-\mathrm{M}}, b / a_{\mathrm{SHV}}, b / a_{\mathrm{TEM}}, b / a_{\mathrm{IMP}}$ and $b / a_{\mathrm{VIM}}$. Real time PCR was carried out in the Light Cycler 2.0 instrument (Roche Applied Science, Germany) using Fast start Light Cycler 480 Hybridization probes Master kit (Roche Diagnostics, USA). Specific primers and probes (Table 1) targeting the genes $b / a_{\mathrm{CTX}-\mathrm{M}}, b / a_{\mathrm{SHV}}, b / a_{\mathrm{TEM}}, b / a_{\mathrm{IMP}}$ and $b / a_{\mathrm{VIM}}$ were amplified by singleplex rPCR. Primers were designed by TIB-Molbiol (Berlin, Germany). rPCR assay was performed in a 32 carousels using $20 \mu \mathrm{L}$ capillaries with each capillary containing a total volume of $20 \mu \mathrm{L}$ including $2 \mu \mathrm{L}$ of Light Cycler FastStart DNA Master Hybridization Probe, $2 \mu \mathrm{L}$ of primers (0.5mM for each forward and reverse), $2.4 \mu \mathrm{L}$ of $\mathrm{MgCl} 2,2 \mu \mathrm{L}$ of extracted DNA, and water to make up the volume of $20 \mu \mathrm{L}$. DNA amplification was carried out with the following run conditions: Pre-incubation for 5 minutes at $95^{\circ} \mathrm{C}$, followed by 45 cycles of amplification with denaturation at $95^{\circ} \mathrm{C}$ for 30 seconds, annealing and extension for 1 minute at $60^{\circ} \mathrm{C}$ without the third step, and then a single cycle of cooling for 30 seconds at $40^{\circ} \mathrm{C}$ [18]. Absolute quantification was carried out using the Light Cycler software 4.05. Positive control strains used include Escherichia coli NCTC 13461 (CTX-M). Klebsiella pneumoniae ATCC 700603 (SHV), Escherichia coli NCTC 13351 (TEM). P. aeruginosa NCTC 13437 (VIM), Escherichia coli NCTC 13476 (IMP). These were obtained from the National Institute of Communicable Diseases (NICD), Johannesburg, South Africa.

Statistical analysis

The data was coded and entered into a database on an Excel spreadsheet and analyzed using Statistical Package for the Social Sciences (SPSS) version 23.0. The descriptive analysis was performed to calculate the frequency and categorical variables were expressed as proportions (\%). All statistical analysis was done with statistical significance set at $\leq 0.05$. 
Table 1

Primer sequences for detection of bla $\mathrm{C}_{\mathrm{CTX}-\mathrm{M}}$, $\mathrm{bla}_{\mathrm{SHV}}$, bla $_{\mathrm{TEM}}$, bla $_{\text {IMP, }}$, bla $\mathrm{a}_{\mathrm{VIM}}$ genes and gyrB

\begin{tabular}{|c|c|c|c|c|}
\hline $\begin{array}{l}\text { Target } \\
\text { gene }\end{array}$ & Primers & Primers sequences $\left(5^{\prime}-3^{\prime}\right)$ & $\begin{array}{l}\mathrm{Tm} \text { in } \\
0^{\circ} \mathrm{C}\end{array}$ & References \\
\hline \multirow[t]{3}{*}{ bla $_{\text {CTX-M }}$} & $\begin{array}{l}\text { CTX-M } \\
\text { forward primer }\end{array}$ & ATGAGYACCAGTAARGTKATGGC & 58.7 & 36 \\
\hline & $\begin{array}{l}\text { CTX-M reverse } \\
\text { primer }\end{array}$ & ATCACKCGGRTCGCCIGGRAT & 59.3 & \\
\hline & CTX-M Probe & FAM-CCCGACAGCTGGGAGACGAAACGT-BBQ & 70.2 & \\
\hline \multirow[t]{3}{*}{ bla $_{\mathrm{SHV}}$} & $\begin{array}{l}\text { SHV forward } \\
\text { primer }\end{array}$ & TCCCATGATGAGCACCTTTAAA & 56.8 & 37 \\
\hline & $\begin{array}{l}\text { SHV reverse } \\
\text { primer }\end{array}$ & TCCTGCTGGCGATAGTGGAT & 58.6 & \\
\hline & SHV Probe & FAM-TGCCGGTGACGAACAGCTGGAG-BBQ & 68.3 & \\
\hline \multirow[t]{3}{*}{$\mathrm{bla}_{\mathrm{TEM}}$} & $\begin{array}{l}\text { TEM forward } \\
\text { primer }\end{array}$ & GCATCTTACGGATGGCATGA & 56.6 & 37 \\
\hline & $\begin{array}{l}\text { TEM reverse } \\
\text { primer }\end{array}$ & GTCCTCCGATCGTTGTCAGAA & 57.7 & \\
\hline & TEM Probe & FAM-CAGTGCTGCCATAACCATGAGTGA-BHQ1 & 62.2 & \\
\hline \multirow[t]{3}{*}{ bla $_{\text {IMP }}$} & $\begin{array}{l}\text { IMP forward } \\
\text { primer }\end{array}$ & GGGCGGAATAGAGTGGCTTA & 57.6 & 38 \\
\hline & $\begin{array}{l}\text { IMP reverse } \\
\text { primer }\end{array}$ & GGCTTGAACCTTACCGTCTTTTT & 59.3 & \\
\hline & IMP Probe & $\begin{array}{l}\text { FAM- } \\
\text { CGATCTATCCCCACGTATGCATCTGAATTAACA- } \\
\text { BHQ1 }\end{array}$ & 67.4 & \\
\hline \multirow[t]{3}{*}{ bla $_{\mathrm{VIM}}$} & $\begin{array}{l}\text { VIM forward } \\
\text { primer }\end{array}$ & TGCGCTTCGGTCCAGTAGA & 59.0 & 38 \\
\hline & $\begin{array}{l}\text { VIM reverse } \\
\text { primer }\end{array}$ & TGACGGGACGTATACAACCAGAT & 58.5 & \\
\hline & VIM Probe & $\begin{array}{l}\text { FAM-CTTCTATCCTGGTGCTGCGCATTCG- } \\
\text { BHQ1 }\end{array}$ & 67.6 & \\
\hline \multirow[t]{2}{*}{ gyrB } & $\begin{array}{l}\text { gyrB forward } \\
\text { primer }\end{array}$ & CCT GAC CAT CCG TCG CCA CAA & & 39 \\
\hline & $\begin{array}{l}\text { gyrB reverse } \\
\text { primer }\end{array}$ & CGC AGC AGG ATG CCG ACG CC & & \\
\hline
\end{tabular}




\begin{tabular}{|c|c|c|c|c|}
\hline $\begin{array}{l}\text { Target } \\
\text { gene }\end{array}$ & Primers & Primers sequences $\left(5^{\prime}-3^{\prime}\right)$ & $\begin{array}{l}\mathrm{Tm} \text { in } \\
0^{\circ} \mathrm{C}\end{array}$ & References \\
\hline & gyrB probe & $\begin{array}{l}\text { 6-FAM-CCG TGG TGG TAG ACC TGT TCC CAG } \\
\text { ACC-BHQ } \\
\text { 6-FAM-CCG TGG TGG TAG ACC TGT TCC CAG } \\
\text { ACC-BBQ }\end{array}$ & & This study \\
\hline
\end{tabular}

\section{Results}

Identification of P. aeruginosa and demographics

During the study period, a total of $204 \mathrm{P}$. aeruginosa isolates were recovered from a range of clinical specimens of patients hospitalized in various wards of NMAH, Mthatha Regional hospital, Secondary district hospitals and primary health care clinics in Mthatha and other towns in four District municipalities of the Eastern Cape Province. The isolates were confirmed by Vitek 2 system (bioMérieux, Inc., USA), Microscan autoscan-4 system (Beckman Coulter, Inc. USA) and rPCR using specific primer and probes targeting gyrB [39]. The majority of the isolates were recovered from male patients (60\%) while $40 \%$ belonged to female patients. The isolates were predominantly from pus and wound swabs $(80.4 \%)$ while the samples originated from Surgical (33.3\%), General (18.1\%) and Paediatrics (11.3\%) wards. 
Table 2

General characteristics of patients and specimen from whose isolates were recovered

\begin{tabular}{|ll|}
\hline Variable & Number, $\mathbf{n}$ (\%) \\
\hline Gender & $122(59.8)$ \\
\hline Male & $82(40.2)$ \\
\hline Female & \\
\hline Age (Years) (mean \pm SEM) & \\
\hline$\leq 15(7.4 \pm 0.6)$ & $44(21.6)$ \\
\hline $16-30(23.9 \pm 0.7)$ & $48(23.5)$ \\
\hline $31-45(37.2 \pm 0.6)$ & $44(21.6)$ \\
\hline $46-60$ (54.1 \pm 0.8$)$ & $30(14.7)$ \\
\hline$>60$ (71.7 \pm 1.3$)$ & $21(10.3)$ \\
\hline Specimen source & \\
\hline Pus and wound swab & $164(80.4)$ \\
\hline Sputum & $22(10.8)$ \\
\hline Fluid aspirate & $7(3.4)$ \\
\hline Catheter tip & $6(2.9)$ \\
\hline Urine & $3(1.5)$ \\
\hline Tissue & $2(1.0)$ \\
\hline MDR status & $12(54(80.4)$ \\
\hline MDR & $75(36.8)$ \\
\hline Non-MDR & $129(63.2)$ \\
\hline District Municipality & \\
\hline Alfred Nzo & \\
\hline Amathole & \\
\hline Joe Gqabi & \\
\hline OR Tambo & \\
\hline
\end{tabular}


The mean age of patients was 32.8 years ranging from 6 days to 84 years. The male population have a mean age of 30.5 years ranging from 6 days to 83 years while the female mean age was 36.2 years with age ranging from 22 days to 84 years. These patients were drawn from four district municipalities with OR Tambo having the most at $80.4 \%$ with the least patient drawn from Joe Gqabi at $0.5 \%$ (Table 2)

\section{Antimicrobial Susceptibility}

Out of 204 isolates tested to various antibiotics, high antibiotic resistance in decreasing order was observed in piperacillin (64,2\%), aztreonam (57.8\%), cefepime (51.5\%), ceftazidime $(51.0 \%)$, piperacillin/tazobactam (50.5\%), and imipenem (46.6\%). Percentages of low resistance in reducing order were observed in gentamicin (35.3\%), meropenem (24.0\%), amikacin (20.1\%), levofloxacin (19.1\%), ciprofloxacin (11.3\%), doripenem (11.3\%), and tobramycin (8.3\%) (Fig. 1) The study also revealed a total of seventy-five isolates $(36.8 \%)$ were multidrug resistant out of the tested isolates while non-MDR constituted $63.2 \%$ of the total.

Molecular Detection Of Esbl- And Mbl-encoding Genes

Of 204 P. aeruginosa isolates, 82 were tested by singleplex rPCR for molecular detection of ESBL and MBL. These data revealed that ESBL- genotypic resistance is driven in Mthatha by bla $\mathrm{TEM}_{\mathrm{TEM}}(79.3 \%)$ followed by bla $\mathrm{SHV}_{\mathrm{SH}}(69.5 \%)$ and lastly bla $\mathrm{CTX}_{\mathrm{CT}}$ (31.7\%). MBL-genotypic resistance, bla $\mathrm{VIM}_{\mathrm{VI}}$, was not detected in all isolated tested while only one bla $\mathrm{IMP}_{\mathrm{MP}}(1.25 \%)$ was detected in all the tested isolates (Table 3) The most common ESBL-genotype combination among the P. aeruginosa was a combination of bla $_{\mathrm{TEM}}+$ bla $_{\mathrm{SHV}}$ at $49(40.5 \%)$. 
Table 3

ESBL genotypes in P. aeruginosa isolates

\begin{tabular}{|lll|}
\hline Positive by PCR for ESBL genes & Number amplified (N = 82) & Total (\%) \\
\hline A. Single ESBL gene & & 79.3 \\
\hline bla $_{\mathrm{TEM}}$ only & 65 & 69.5 \\
\hline bla $_{\mathrm{SHV}}$ only & 57 & 31.7 \\
\hline bla $_{\mathrm{CTX}-\mathrm{M}}$ only & 26 & 1.25 \\
\hline bla $_{\mathrm{IMP}}$ only & 1 & 0 \\
\hline bla $_{\mathrm{VIM}}$ only & 0 & 40.5 \\
\hline B. TWO or more ESBL genes & 121 & 18.2 \\
\hline bla $_{\mathrm{TEM}}+$ bla $_{\mathrm{SHV}}$ & 49 & 21.5 \\
\hline bla $_{\mathrm{TEM}}+$ bla $_{\mathrm{CTX}-\mathrm{M}}$ & 22 & 0.8 \\
\hline bla $_{\mathrm{SHV}}+$ bla $_{\mathrm{CTX}-\mathrm{M}}$ & 26 & 18.2 \\
\hline bla $_{\mathrm{TEM}}+$ bla $_{\text {IMP }}$ & 1 & 0.8 \\
\hline bla $_{\mathrm{TEM}}+$ bla $_{\mathrm{SHV}}+$ bla $_{\mathrm{CTX}-\mathrm{M}}$ & 22 & 0 \\
\hline bla $_{\mathrm{TEM}}+$ bla $_{\mathrm{SHV}}+$ bla $_{\text {IMP }}$ & 1 & \\
\hline bla $_{\mathrm{TEM}}+$ bla $_{\mathrm{SHV}}+$ bla $_{\mathrm{CTX}-\mathrm{M}}+\mathrm{bla}_{\mathrm{IMP}}$ & 0 & \\
\hline
\end{tabular}

In ESBL-producing P. aeruginosa the highest level of resistance was observed against piperacillin (52.7\%), followed by aztreonam (47.3\%), ceftazidime (44.6\%), cefepime $(41.9 \%)$ and imipenem and piperacillin/tazobactam (both at 40.5\%); while in non-ESBL-producing isolates, highest level of resistance was observed to gentamicin and ceftazidime (62.5\%) and low resistance level to levofloxacin $(12.5 \%)$ (Table 4). 
Table 4

Resistance pattern of ESBL and non-ESBL Pseudomonas aeruginosa isolates

\begin{tabular}{|llll|}
\hline Antibiotics & $\begin{array}{l}\text { Total }(\mathbf{N}=82) \\
\text { No }(\%)\end{array}$ & $\begin{array}{l}\text { ESBL }(\mathbf{N}=74) \\
\text { No }(\%)\end{array}$ & $\begin{array}{l}\text { Non-ESBL (N=8) } \\
\text { No }(\%)\end{array}$ \\
\hline Amikacin & $26(31.7)$ & $23(31.1)$ & $3(37.5)$ \\
\hline Aztreonam & $38(46,3)$ & $35(47.3)$ & $3(37.5)$ \\
\hline Cefepime & $37(45.1)$ & $31(41.9)$ & $6(75.0)$ \\
\hline Ceftazidime & $38(46.3)$ & $33(44.6)$ & $5(62.5)$ \\
\hline Ciprofloxacin & $15(18.3)$ & $13(17.6)$ & $2(25.0)$ \\
\hline Doripenem & $17(20.7)$ & $17(23,0)$ & $0(0.0)$ \\
\hline Gentamicin & $33(40.2)$ & $28(37.8)$ & $5(62.5)$ \\
\hline Imipenem & $33(40.2)$ & $30(40.5)$ & $3(37.5)$ \\
\hline Levofloxacin & $16(19.5)$ & $15(20.3)$ & $1(12.5)$ \\
\hline Meropenem & $27(32,9)$ & $25(33.8)$ & $2(25.0)$ \\
\hline Piperacillin & $43(52.4)$ & $39(52.7)$ & $4(50.0)$ \\
\hline Piperacillin/tazobactam & $34(41,5)$ & $30(40.5)$ & $4(50.0)$ \\
\hline Tobramycin & $16(19.5)$ & $13(17.6)$ & $3(37.5)$ \\
\hline
\end{tabular}

Different parameters were tested in relationship to the production of ESBL by IPCR in our tested isolates. The samples were drawn from both secondary district hospitals and tertiary level of hospital setting. No sample was recovered from the primary health care center. Our data revealed that the hospital type had no significant effect on the acquisition of ESBL and that the effect of tertiary hospital on ESBL is $43 \%$ lesser than the secondary hospital (OR: 0.57; Cl: 0.132-2.49; P:0.458). (Table 5).

The relationship between specimen type and ESBL production revealed that sputum was found to be associated with ESBL-producing P. aeruginosa at $95 \%$ confidence interval range of $0.03-0.92$ and OR of 0.16 (Table 6). 
Table 5

Multivariate analysis of factors associated with ESBL-positive and ESBLnegative isolates

\begin{tabular}{|lllll|}
\hline Variable & $\begin{array}{l}\text { ESBL } \\
\text { No }(\%)\end{array}$ & $\begin{array}{l}\text { Non-ESBL } \\
\text { No }(\%)\end{array}$ & OR (95\% Cl) & p-value \\
\hline Gender & & & & \\
\hline Male & $49(91)$ & $5(9)$ & & \\
\hline Female & $25(89)$ & $3(11)$ & $0.85(0.187-3.90)$ & 0.833 \\
\hline Hospital type & & & & \\
\hline Secondary & $47(92.2)$ & $4(7.8)$ & $0.57(0.132-2.49)$ & 0.458 \\
\hline Tertiary & $27(87.1)$ & $4(12.9)$ & & \\
\hline
\end{tabular}

Table 6

Relationship between ESBL and selected parameters (Adjusted OR)

\begin{tabular}{|c|c|c|c|c|}
\hline Variables & & OR (95\% Cl) & $\mathrm{Cl}$ & $\mathbf{P}$ \\
\hline \multirow[t]{2}{*}{ Hospital } & Secondary & 1 & & \\
\hline & Tertiary & 0.574 & $0.132-2.48$ & 0.458 \\
\hline \multirow[t]{4}{*}{ Age } & Less 15 years & 1 & & \\
\hline & $16-30$ & 0.68 & $0.08-5.49$ & 0.721 \\
\hline & $31-45$ & 1.10 & $0.14-8.63$ & 0.924 \\
\hline & 46 and more & 1 & & \\
\hline \multirow[t]{2}{*}{ Gender } & Male & 1 & & \\
\hline & Female & 0.85 & $0.18-3.85$ & \\
\hline \multirow[t]{4}{*}{ Specimen } & Pus and wound & 1 & & \\
\hline & Sputum & 0.16 & $0.03-0.92$ & 0.040 \\
\hline & Fluid & 0.11 & $0.07-1.54$ & 0.101 \\
\hline & Urine & 0.11 & $0.07-1.54$ & 0.101 \\
\hline
\end{tabular}

\section{Discussion}

The current study revealed a moderate to high antibiotic resistance in decreasing order was observed in piperacillin (64.2\%), aztreonam (57.8\%), Cephalosporins (cefepime 51.5\% and ceftazidime $51.0 \%$ ), 
antipseudomonal penicillins $+\beta$-lactamase inhibitor (piperacillin/tazobactam 50.5\%) and imipenem (46.6\%). (Fig. 1). Low resistance in reducing order were observed in gentamicin (35.3\%), meropenem (24.0\%), amikacin (20.1\%), fluoroquinolones (levofloxacin 19.1\% and ciprofloxacin 11.3\%), doripenem (11.3\%), and tobramycin (8.3\%). Data from surveillance on isolates of P. aeruginosa in the South African private laboratories is not in agreement with our study [40]. They reported a much lower resistance rates of $28.2 \%, 26.3 \%, 31.9 \%, 37.8 \%, 35.5 \%$ and $35.5 \%$ in cefepime, ceftazidime, doripenem, imipenem, meropenem and piperacillin respectively. The isolates were recovered from blood culture only, possibly this might account for the difference in resistance rate, alternatively this might be due to regional variations in the empirical use of these antimicrobials [40]. In our study, the percentage of resistance of $11.3 \%$ to ciprofloxacin was within the same range of $13.4 \%$ described by Ramsamy et al., [28]. The data obtained were from nine public sector hospitals in Kwazulu-Natal Province. Additionally, gentamicin resistance of $17 \%$ and imipenem resistance of $13 \%$ as reported in the study was lower in comparison with resistance reported in our study at $35.3 \%$ and $46.6 \%$ respectively. The susceptibility ranges of $75 \%-92 \%$ of P. aeruginosa isolates in this study to routine antibiotics considered for therapy is encouraging but the increase in resistance exhibited to cephalosporins and imipenem is concerning. This might be due to selective pressure to those antibiotics and it will be important to monitor the prescription of these antibiotics. Owing to endless alteration, resistance exhibited to range of $\beta$-lactam antibiotics is challenging, thus making $\beta$-lactamase production the commonest cause of drug resistance and antimicrobial treatment tragedy $[10,41]$. Our study detected an average resistance of $45.7 \%$ to cephalosporins (ceftazidime and cefepime) in ESBL P. aeruginosa. Piperacillin and gentamicin resistance in ESBL isolates was $52.4 \%$ and $40.2 \%$ respectively (Table 4) which is similar to the findings of Farhan et al., [10]. The emerging level of resistance displayed to the cephalosporins highlight the development of cephalosporinases among resistant strains of these organisms. The cephalosporins due to their wide spectrum of activity are a significant class of antimicrobials used in controlling several infections however, the emergence of cephalosporinases can in effect hamper their clinical usefulness [42]. The reported increasing penicillinase-producing $\beta$-lactamases strains among these organisms validates the noticeably observable high rate resistance of our isolates to piperacillin [42]. Antibiotic resistance is a public health menace with an alarming proportion that is getting collective attention more so that several studies have found a correlation between level of antibiotic prescription with the prevalence of antibiotic resistance [43-44]. Patients with resistant P. aeruginosa infections have a poor prognosis hence it is imperative that $P$. aeruginosa strains presenting severe drug resistance is monitored [23]. The swift spread and the emergence of MBL- and ESBL-producing P. aeruginosa of clinical origin is distressing and of great threat. Furthermore, level of antibiotic usage, horizontal gene transfer (HGT) event and environmental factors may account for variations in resistance patterns among strains isolated from diverse countries and regions. Antimicrobial susceptibility testing and proper screening for ESBL and MBL production has to be embarked upon before antimicrobial therapy [10]. In the present study, 36.8\% of our isolates were MDR (defined as non-susceptibility to at least one agent in three or more antimicrobial categories). Similarly, MDR rate of $45 \%$ was reported by Fazeli et al., [45] however Sahoo et al., [46] reported a higher rate of resistance at $72.69 \%$. 
The emergence of ESBL-producing P. aeruginosa is increasingly reported as a major cause of health-care associated infections. In the hospital locale, infections resulting from these resistant organisms are increasingly challenging to treat due to the intensity of resistance exhibited to the most commonly recommended antibiotics. Antimicrobial treatment is further hampered by the production of extended spectrum beta-lactamases and metallo beta-lactamases [20,47]. As at now, ESBLs in P. aeruginosa are described globally with MBLs also being reported on a growing basis in recent years $[15,22]$. Various studies have reported the existence of ESBLs in clinical isolates of P. aeruginosa $[16,19,48]$. Class $A$ ESBLs are typically recognized in $\mathrm{P}$. aeruginosa isolates presenting resistance to extended-spectrum cephalosporin. Traditional ESBLs have developed from restricted-spectrum class A TEM and SHV $\beta$ lactamases although a variety of non-TEM and non-SHV class A ESBLs have been described including CTX-M, PER, VEB, GES, and BEL [49]. The ESBL genotype differs in various parts of the world. This study found out that the most prevalent genotype for ESBL production was bla TEM $_{\text {which }}$ was detected in 65 (79.3\%) isolates followed by bla $\mathrm{SHV}_{\text {at }} 57$ (69.5\%) and bla $\mathrm{CTX}_{\mathrm{M}}$ at 26 (31.7\%) (Table 3) Since it has been reported that ESBL genes show variation depending on the geographical location, the findings of Erhlers et al., [50], Chen et al., [15], Miranda et al., [51], Bokaeian et al., [52] and Khurana et al., [20] in South Africa, China, Brazil, Iran and India respectively corroborated the prevalent genotype to be bla ${ }_{\mathrm{TEM}}$ while contrarily Jamali et al., [53] and Rezai et al., [54] reported the prevalent gene to be $\mathrm{bla}_{\mathrm{SHV}}$ at $75 \%$ and $86.66 \%$ respectively. The predominant ESBL and MBL genes detected in a study conducted in Durban on MDR P. aeruginosa isolates were GES-2, OXA-21, and VIM-2 [55]. The least detected ESBL genotype was CTX-M in $26(31.7 \%)$ isolates similar to Miranda et al., [51]. The MBL genotype, bla IMP $_{\text {was }}$ whly detected in 1 sample (1.25\%) while bla ${ }_{V I M}$ was not detected in any of the sample. Similar findings were reported in Egypt by Abbas et al., [56], where no VIM genes was detected by PCR analysis. In contrast, Zafer et al., and Adjei et al., $[49,55]$ reported a prevalence of $58.2 \%$ and $29 \%$ respectively in VIM-2. MDR P. aeruginosa encoding bla $\mathrm{VIM}_{\mathrm{V}-2}$ gene have been reported in a tertiary hospital in Cape Town, which was responsible for an outbreak, and in a public hospital in Port Elizabeth [57, 58]. The study of Arunagiri et al., [59] reported low rate of bla $_{\text {IMP }}$ in $2(3 \%)$ isolates which is similar to our study. The phenotypic resistance displayed to the carbapenems particularly imipenem which is not validated by the genotypic MBL result may be due to other resistance mechanism such as efflux over expression or forfeiture of exterior membrane protein [60, 61].

Several researchers have reported on the concurrence of different $\beta$-lactamase genes found in the same isolates $[46,49,62]$. The most common ESBL combination among our isolates by genotype was a combination of bla $\mathrm{TEM}_{\mathrm{TEM}}+$ bla $_{\mathrm{SHV}}$ at 49 (40.5\%) but contrary to Chen et al., [15] and Sahoo et al., [46], who

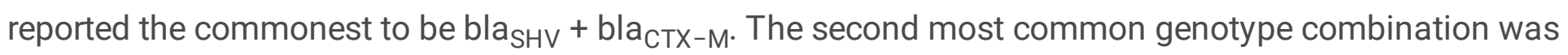
a combination of bla SHV + bla $_{\text {CTX-M }}$ which is similar to the study from Syria and Philippines [63, 64]. Our study showed the most predominant ESBL gene to be TEM which was corroborated by other studies. Prior to now, TEM used to be the most prevalent but recent reports suggest that the CTX-M-type group of ESBLs may now be the most predominant type globally $[46,65]$. 


\section{Conclusion}

Our study is the first surveillance report on molecular characteristics and antimicrobial susceptibility testing of P. aeruginosa isolates from clinical samples of patients in Mthatha, Eastern Cape Province South Africa. The study showed a high rate of MDR P. aeruginosa in our setting. The vast majority of these resistant isolates carried bla $\mathrm{TEM}_{\mathrm{TEM}}$ and bla $\mathrm{SHV}_{\mathrm{SV}}$ genes. Fortunately, we did not detect any bla $\mathrm{VIM}_{\mathrm{M}}$ in all the tested isolates with bla $\mathrm{IMP}_{\mathrm{MP}}$ reported as just one. ESBLs are becoming progressively multifaceted and diverse. Early detection and characterization is critical to contain their dissemination, prevent outbreak and enhance intervention strategies and optimise therapy. Continuous monitoring of antimicrobial resistance and strict compliance towards infection control practices are the best defence against continuous spread of MDR P. aeruginosa.

\section{Abbreviations}

ESBL: extended-spectrum beta-lactamase; MBL: metallo- $\beta$-lactamases; AST: antimicrobial susceptibility testing; rPCR: Real-time polymerase chain reaction; MDR: multidrug-resistant; HGT: horizontal gene transfer; NMAH: Nelson Mandela Academic Hospital; NHLS: National Health Laboratory Services; NICD: National Institute of Communicable Diseases; CLSI: Clinical Laboratory Standards Institute; WHO: World Health Organization; CDC: Centre for Disease Prevention and Control.

\section{Declarations}

Authors' contributions

M.C.H., T.A., S.V Conceived and designed the experiment. M.C.H., S.D. performed the experiments: M. C. H., T. A. analysed the data: M.C.H. wrote the manuscript: M.C.H., T.A., S.V., G.E.O analysis and interpretation of data; proof reading of manuscript. All authors read and approved the final manuscript

Authors' details

${ }^{1}$ Division of Medical Microbiology, Department of Laboratory Medicine and Pathology, Faculty of Health Sciences, Walter Sisulu University, Private Bag X1, and National Health Laboratory Services, Mthatha, Eastern Cape 5117, South Africa. ${ }^{2}$ Department of Biological and Environmental Sciences, Faculty of Natural Sciences, Walter Sisulu University, Private Bag X1, Mthatha, Eastern Cape 5117, South Africa

\section{Funding}

The authors declare that they did not have any funding source or grant to support their research work.

Acknowledgements

The authors are grateful to the Research Directorate of the Walter Sisulu University. The Nelson Mandela Academic Hospital (NMAH) and the Microbiology Laboratory at the National Health Laboratory Services 
(NHLS) at NMAH are also acknowledged. The National Institute of Communicable Diseases (NICD) is appreciated for supply of reference strains. The effort of Dr. Ndebia J. in data analysis is highly appreciated as well.

Competing interests

The authors declare that they have no competing interests.

Availability of data and materials

All data generated or analysed during this study are included in this published article.

Ethics approval and consent to participate

Ethical approval for the study was granted by the Health Research Ethics and Biosafety Committee of the Faculty of Health Sciences, Walter Sisulu University (WSU) Reference number 024/2016 while permission to conduct the study was obtained from the National Health Laboratory Services (NHLS).

Consent for publication

Not applicable

\section{References}

1. Breidenstein EB, de la Fuente-Núñez C, Hancock RE. Pseudomonas aeruginosa: all roads lead to resistance. Trends Microbiol. 2011;19(8):419-26.

2. Henrichfreise B, Wiegand I, Pfister W, Wiedemann B. Resistance mechanisms of multiresistant Pseudomonas aeruginosa strains from Germany and correlation with hypermutation. Antimicrob Agents and Chemother. 2007;51(11):4062-70.

3. Yayan J, Ghebremedhin B, Rasche K. Antibiotic Resistance of Pseudomonas aeruginosa in Pneumonia at a Single University Hospital Center in Germany over a 10-Year Period. PLoS ONE. 2015; 10(10): e0139836.

4. Subedi D, Vijay AK, Kohli GS, Rice SA, Willcox M. Comparative genomics of clinical strains of Pseudomonas aeruginosa strains isolated from different geographic sites. Sci Rep. 2018; 23;8(1):14.

5. Ciofu O, Tolker-Nielsen T. Tolerance and resistance of Pseudomonas aeruginosa biofilms to antimicrobial agents - how aeruginosa can escape antibiotics. Front Microbiol. 2019; 10:913.

6. de Melo AC, da Mata Gomes A, Melo FL, Ardisson-Araújo DM, de Vargas AP, Ely VL, Kitajima EW, Ribeiro BM, Wolff JL. Characterization of a bacteriophage with broad host range against strains of Pseudomonas aeruginosa isolated from domestic animals. BMC Microbiology. 2019;19(1):134.

7. Rocha AJ, Barsottini MR, Rocha RR, Laurindo MV, Moraes FL, Rocha SL. Pseudomonas Aeruginosa: Virulence Factors and Antibiotic Resistance Genes. Brazilian Archives of Biology and Technology. 
2019;62.

8. Frieden T. Antibiotic resistance threats in the United States, 2013. Centers for Disease Control and Prevention, US Department of Health and Human Services. 2013; 23:11-28.

9. Tacconelli E, Magrini N, Kahlmeter G, Singh N. Global priority list of antibiotic-resistant bacteria to guide research, discovery, and development of new antibiotics. World Health Organization. $2017 ; 27$.

10. Farhan SM, Ibrahim RA, Mahran KM, Hetta HF, El-Baky RM. Antimicrobial resistance pattern and molecular genetic distribution of metallo- $\beta$-lactamases producing Pseudomonas aeruginosa isolated from hospitals in Minia, Egypt. Infect Drug Resist. 2019; 12:2125.

11. Hwang W, Yoon SS. Virulence Characteristics and an Action Mode of Antibiotic Resistance in Multidrug-Resistant Pseudomonas aeruginosa. Sci Rep,2019;9:487

12. Chaudhary M, Payasi A. Rising Antimicrobial Resistance of Pseudomonas aeruginosa Isolated from Clinical Specimens in India. J Proteomics Bioinform, 6(1): 005-9.

13. Laudy AE, Rog P, Smolińska-Król K, Ćmiel M, Słoczyńska A, Patzer J, Dzierżanowska D, Wolinowska R, Starościak B, Tyski S. Prevalence of ESBL-producing Pseudomonas aeruginosa isolates in Warsaw, Poland, detected by various phenotypic and genotypic methods. PloS One. 2017;12(6).

14. Kaur A, Singh S. Prevalence of Extended Spectrum Beta lactamase (ESBL) and Metallobetalactamase (MBL) Producing Pseudomonas aeruginosa and Acinetobacter baumannii Isolated from Various Clinical Samples. J Pathog. 2018;2018.

15. Chen Z, Niu H, Chen G, Li M, Li M, Zhou Y. Prevalence of ESBLs-producing Pseudomonas aeruginosa isolates from different wards in a Chinese teaching hospital. Int J Clin Exp Med. 2015;8(10):19400.

16. Bharti NM, Sharma PC. Molecular characterization of Pseudomonas aeruginosa isolates recovered from human patients in Himachal Pradesh (India) for selective genes: Extended spectrum $\beta$ lactamase (ESBL) Ampicillin Class C (AmpC) and metallo $\beta$ - lactamase (MBL) genes. IJPSR, 2016. 7(12): 4905-4916.

17. Gniadkowski M. Evolution and Epidemiology of Extended-spectrum $\beta$-lactamases (ESBLs) and ESBLproducing microorganisms. Clin Microbiol Infect, 2001 7(11): 597-608.

18. Vasaikar S, Obi L, Morobe I, Bisi-Johnson M. Molecular characteristics and antibiotic resistance profiles of Klebsiella isolates in Mthatha, Eastern Cape province, South Africa. Int. J. Microbiol. 2017;2017.

19. Founou RC, Founou LL, Essack SY. Extended spectrum beta-lactamase mediated resistance in carriage and clinical gram-negative ESKAPE bacteria: a comparative study between a district and tertiary hospital in South Africa. Antimicrob Resist Infect Control, 2018; 7:134.

20. Khurana S, Mathur P, Kapil A, Valsan C, Behera B. Molecular epidemiology of beta-lactamase producing nosocomial Gram-negative pathogens from North and South Indian hospitals. J Med Microbiol. 2017; 66: 999-1004.

21. Alfaresi MS, Elkoush AA. Real-time polymerase chain reaction for rapid detection of genes encoding SHV extended-spectrum $\beta$-lactamases. Indian J Med Microbiol, 2010 ,28(4): 332-336. 
22. Lin SP, Liu MF, Lin CF, Shi ZY. Phenotypic detection and polymerase chain reaction screening of extended-spectrum $\beta$-lactamases produced by Pseudomonas aeruginosa J Microbiol, Immunol Infect. 2012;45(3):200-7.

23. Dou Y, Huan J, Guo F, Zhou Z, Shi Y. Pseudomonas aeruginosa prevalence, antibiotic resistance and antimicrobial use in Chinese burn wards from 2007 to 2014. J Int Med Res. 2017;45(3):1124-37.

24. Piri F, Ebrahimi TM, Amini K. Molecular investigation of CTX-M gene in Extended Spectrum $\beta$ Lactamases (ESBLs) producing Pseudomonas aeruginosa isolated from Iranian patients with burn wound infection. Arch Med Lab Sci. 2018; 4(1): 1-4.

25. Osawa K, Shigemura K, Kitagawa K, Fukuda T, Takasaka A, Wakabayashi S, Sato K, Yamamichi F, Shirakawa T, Fujisawa M. Molecular characteristics of carbapenem-resistant Pseudomonas aeruginosa isolated from urine in Hyogo, Japan. Int J Urol. 2019;26(1):127-33.

26. Poirel L, Weldhagen GF, De Champs C, Nordmann P. A nosocomial outbreak of Pseudomonas aeruginosa isolates expressing the extended-spectrum $\beta$-lactamase GES-2 in South Africa. J Antimicrob Chemother. 2002;49(3):561-5.

27. Mudau M, Jacobson R, Minenza N, Kuonza L, Morris V, Engelbrecht H, Nicol MP, Bamford C. Outbreak of multi-drug resistant Pseudomonas aeruginosa bloodstream infection in the haematology unit of a South African Academic Hospital. PloS One. 2013;8(3).

28. Ramsamy Y. Essack SY, Sartorius B, Patel M, Mlisana KP. Antibiotic resistance trends of ESKAPE pathogens in Kwazulu-Natal, South Africa: A five-year retrospective analysis. Afr J Lab Med. 2018; $7(2), a 887$.

29. Founou RC, Founou LL, Allam M, Ismail A, Essack SY. Whole genome sequencing of extended spectrum $\beta$-lactamase (ESBL)-producing Klebsiella pneumoniae isolated from hospitalized patients in KwaZulu-Natal, South Africa. Sci Rep. 2019; 9(1):1-1.

30. Selim S, El Kholy I, Hagagy N, El Alfay S, Aziz MA. Rapid identification of Pseudomonas aeruginosa by pulsed-field gel electrophoresis. Biotechnol Biotec Eq. 2015; 29(1):152-6.

31. Solomon FB, Wadilo F, Tufa EG, Mitiku M. Extended spectrum and metalo beta-lactamase producing airborne Pseudomonas aeruginosa and Acinetobacter baumanii in restricted settings of a referral hospital: a neglected condition. Antimicrob Resist Infect Control. 2017; 6(1):106.

32. Govender N, Perovic O. GERMS-SA Laboratory-based Surveillance for Antimicrobial-resistant Bacterial and Fungal Bloodstream Infections, 2016.

33. Lutgring JD, Kim A, Campbell D, Karlsson M, Brown AC, Burd EM. Evaluation of the MicroScan colistin well and gradient diffusion strips for colistin susceptibility testing in Enterobacteriaceae. $J$ Clin Microbiol. 2019;57(5): e01866-18.

34. Clinical and Laboratory Standards Institute (CLSI). Performance Standards for Antimicrobial Susceptibility Testing; 20th Informational Supplement. CLSI document M100-S20, 2010. CLSI, Wayne, PA: Clinical and Laboratory Standards Institute.

35. Magiorakos AP, Srinivasan A, Carey RB, Carmeli Y, Falagas ME, Giske CG, Harbarth S, Hindler JF, Kahlmeter G, Olsson-Liljequist B, Paterson DL. Multidrug-resistant, extensively drug-resistant and 
pandrug-resistant bacteria: an international expert proposal for interim standard definitions for acquired resistance. Clin Microbiol Infect. 2012;18(3):268-81.

36. Angeletti S, Gherardi G, De Florio L, Avola A, Crea F, Riva E, Vitali MA, Galluzzo S, Dicuonzo G. Realtime polymerase chain reaction with melting analysis of positive blood culture specimens in bloodstream infections: diagnostic value and turnaround time. New Microbiol. 2013; 36:65-74.

37. Roschanski N, Fischer J, Guerra B, Roesler U. Development of a multiplex real-time PCR for the rapid detection of the predominant beta-lactamase genes CTX-M, SHV, TEM and CIT-type AmpCs in Enterobacteriaceae. PloS One. 2014;9(7).

38. Wendel AF, Brodner AH, Wydra S, Ressina S, Henrich B, Pfeffer K, Toleman MA, MacKenzie CR. Genetic characterization and emergence of the metallo- $\beta$-lactamase GIM-1 in Pseudomonas and Enterobacteriaceae during a long-term outbreak. Antimicrob Agents Chemother. 2013;57(10):5162-5.

39. Anuj SN, Whiley DM, Kidd TJ, Bell SC, Wainwright CE, Nissen MD, Sloots TP. Identification of Pseudomonas aeruginosa by a duplex real-time polymerase chain reaction assay targeting the ecfX and the gyrB genes. Diagn Microbiol Infect Dis. 2009;63(2):127-31.

40. Perovic O, Ismail H, Van Schalkwyk E, Lowman W, Prentice E, Senekal M, Govind CN. Antimicrobial resistance surveillance in the South African Private Sector Report for 2016. S Afr J Infect Dis. 2018 Oct 2;33(4):114-7.

41. Pitout JD. Enterobacteriaceae Producing ESBLs in the Community: Are They a Real Threat? Infect Med. 2007; 24(2):57-65.

42. Osundiya OO, Oladele RO, Oduyebo 00. Multiple Antibiotic Resistance (MAR) Indices of Pseudomonas and Klebsiella species in Lagos Teaching Hospital. Afr. J. Cln. Exper. Microbiol. 2013; 14(3): 164-168.

43. Goldstein E, MacFadden DR, Lee RS, Lipsitch M. Outpatient prescribing of four major antibiotic classes and prevalence of antimicrobial resistance in US adults. bioRxiv. 2018:456244.

44. Sanz-García F, Hernando-Amado S, Martínez JL. Mutational Evolution of Pseudomonas aeruginosa Resistance to Ribosome-Targeting Antibiotics. Front. Genet. 2018; 9:451.

45. Fazeli H, Sadighian H, Esfahani BN, Pourmand MR. Genetic characterization of Pseudomonas aeruginosa-resistant isolates at the university teaching hospital in Iran. Adv Biomed Res. 2015;4.

46. Sahoo S, Otta S, Swain B, Kar SK. Detection and genetic characterization of extended-spectrum betalactamases producers in a tertiary care hospital. J Lab Physicians, 2019. 11: 253-258.

47. Amirkamali S, Naserpour-Farivar T, Azarhoosh K, Peymani A. Distribution of the bla OXA, bla VEB-1, and bla GES-1 genes and resistance patterns of ESBL-producing Pseudomonas aeruginosa isolated from hospitals in Tehran and Qazvin, Iran. Rev Soc Bras Med Trop. 2017;50(3):315-20.

48. Zeynudin A, Pritsch M, Schubert S, Messerer M, Liegl G, Hoelscher M, Belachew T, Wieser A. Prevalence and antibiotic susceptibility pattern of CTX-M type extended-spectrum $\beta$-lactamases among clinical isolates of gram-negative bacilli in Jimma, Ethiopia. BMC Infect Dis. 2018;18(1):524.

49. Zafer MM, Al-Agamy MH, El-Mahallawy HA, Amin MA, Ashour MS. Antimicrobial resistance pattern and their beta-lactamase encoding genes among Pseudomonas aeruginosa strains isolated from 
cancer patients. BioMed Res Int. 2014;2014.

50. Ehlers MM, Veldsman C, Makgotlho EP, Dove MG, Hoosen AA, Kock MM. Detection of bla ${ }_{\mathrm{SHV}}$, bla $a_{\mathrm{TEM}}$ and bla ${ }_{\text {CTX-M }}$ antibiotic resistance genes in randomly selected bacterial pathogens from the Steve Biko Academic Hospital. FEMS Immunol Med Microbiol. 2009 Aug 1;56(3):191-6.

51. Miranda CC, de Filippis I, Pinto LH, Coelho-Souza T, Bianco K, Cacci LC, Picão RC, Clementino MM. Genotypic characteristics of multidrug-resistant Pseudomonas aeruginosa from hospital wastewater treatment plant in Rio de Janeiro, Brazil. J Appl Microbiol. 2015; 118(6):1276-86.

52. Bokaeian M, Zahedani SS, Bajgiran MS, Moghaddam AA. Frequency of PER, VEB, SHV, TEM and CTX-M genes in resistant strains of Pseudomonas aeruginosa producing extended spectrum $\beta$ lactamases. Jundishapur J Microbiol. 2015;8(1).

53. Jamali S, Shahid M, Sobia F, Singh A, Khan HM. Phenotypic and molecular characterization of cefotaximases, temoniera, and sulfhydryl variable $\beta$-lactamases

in Pseudomonasand Acinetobacterisolates in an Indian tertiary health-care center. Indian J Pathol Microbiol, 2017; 60:196-201.

54. Rezai MS, Ahangarkani F, Rafiei A, Hajalibeig A, Bagheri-Nesami M. Extended-Spectrum BetaLactamases Producing Pseudomonas aeruginosa Isolated From Patients With Ventilator Associated Nosocomial Infection. Arch Clin Infect Dis. 2018;13(4).

55. Adjei CB, Govinden U, Moodley K, Essack SY. Molecular characterisation of multidrug-resistant Pseudomonas aeruginosa from a private hospital in Durban, South Africa. S Afr J Infect Dis. 2018;33(2):38-41.

56. Abbas HA, El-Ganiny AM, Kamel HA. Phenotypic and genotypic detection of antibiotic resistance of Pseudomonas aeruginosa isolated from urinary tract infections. Afri Health Sci, 2018; 18(1): 11-21.

57. Jacobson RK, Minenza N, Nicol M, Bamford C. VIM-2 metallo- $\beta$-lactamase-producing Pseudomonas aeruginosa causing an outbreak in South Africa. J Antimicrob Chemother 2012; 67(7):1797-1798.

58. Govender S, Masunda T, Black J. VIM-2 carbapenemase-producing Pseudomonas aeruginosa in a patient from Port Elizabeth, South Africa. SAMJ: S Afr Med J. 2015 May;105(5):328-.

59. Arunagiri K, Sekar B, Sangeetha G, John J. Detection and characterization of metallo- $\beta$-lactamases in Pseudomonas aeruginosa by phenotypic and molecular methods from clinical samples in a tertiary care hospital. West Indian Med J. 2012;61(8):778-83.

60. Polloto M, Casella T, Oliveria M, Rubio F, Nogueira M, Almeida M. Detection of P. areruginosa harboring blaCTX-M-2, blaGES-1 and blaGES-5, blalMP-1 and blaSPM-1 causing infections in Brazilian tertiary-care hospital. BMC Infect Dis. 2012; 12:176.

61. Lee CH, Su TY, Ye JJ, Hsu PC, Kuo AJ, Chia JH, Lee MH. Risk factors and clinical significance of bacteremia caused by Pseudomonas aeruginosa resistant only to carbapenems. J Microbiol, Immunol Infect. 2017;50(5):677-83.

62. Bahrami M, Mohammadi-Sichani M, Karbasizadeh V. Prevalence of SHV, TEM, CTX-M and OXA-48 $\beta$ Lactamase Genes in Clinical Isolates of Pseudomonas aeruginosa in Bandar-Abbas, Iran. Avicenna J Clin Microbiol Infect. 2018;5(4):86-90. 
63. Ibrahim AS, Youssef N. Prevalence of CTX-M, TEM and SHV Beta-lactamases in Clinical Isolates of Escherichia Coli and Klebsiella Pneumoniae Isolated From Aleppo University Hospitals, Aleppo, Syria. Arch Clin Infect Dis. 2015. 10(2).

64. Gundran RS, Cardenio PA, Villanueva MA, Sison FB, Benigno CC, Kreausukon K, Pichpol D,

Punyapornwithaya V. Prevalence and distribution of bla CTX-M, bla SHV, bla TEM genes in extendedspectrum $\beta$-lactamase-producing coli isolates from broiler farms in the Philippines. BMC Vet Res. 2019;15(1):227.

65. Rawat D, Nair D. Extended-spectrum $\beta$-lactamases in Gram Negative Bacteria. J. Glob Infect Dis. 2010; 2(3): 263-274.

\section{Figures}

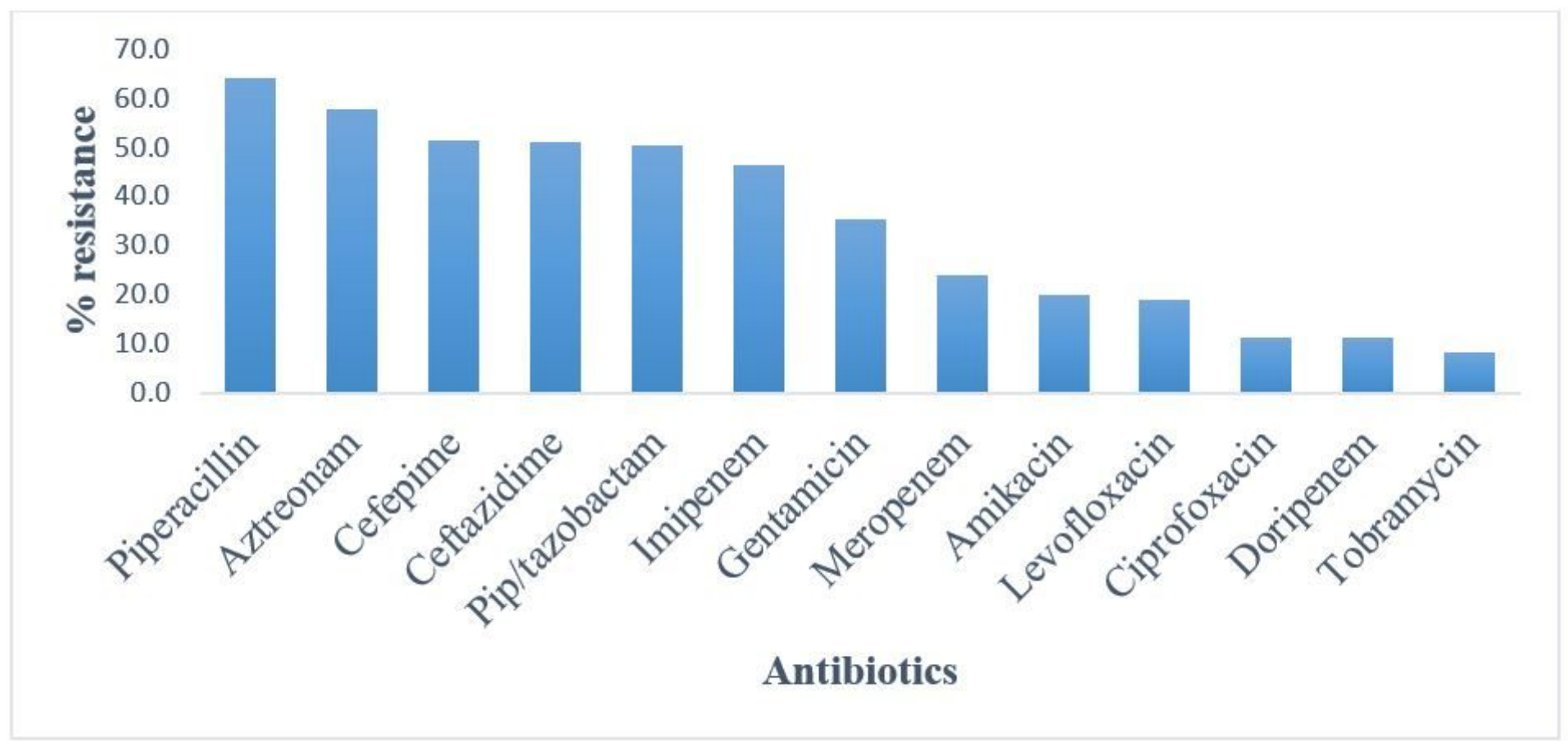

\section{Figure 1}

Antibiotic resistant pattern of the isolates $(\mathrm{N}=204)$ Molecular Detection of ESBL- and MBL-encoding genes 